\title{
The Strong Local Diagnosability of a Hypercube Network with Missing Edges
}

\author{
Min Xie, ${ }^{1,2}$ Jiarong Liang $\mathbb{D}^{2,3}$ and Xi Xiong ${ }^{2,3}$ \\ ${ }^{1}$ School of Automation Science and Engineering, South China University of Technology, 510640, China \\ ${ }^{2}$ School of Computer and Electronics Information, Guangxi University, 530004, China \\ ${ }^{3}$ School of Computer and Electronics Information, Guangxi Key Laboratory of Multimedia Communications and \\ Network Technology, 530004, China
}

Correspondence should be addressed to Jiarong Liang; 13977106752@163.com

Received 15 April 2018; Revised 5 July 2018; Accepted 13 August 2018; Published 4 October 2018

Academic Editor: Michele Scarpiniti

Copyright (c) 2018 Min Xie et al. This is an open access article distributed under the Creative Commons Attribution License, which permits unrestricted use, distribution, and reproduction in any medium, provided the original work is properly cited.

\begin{abstract}
In the research on the reliability of a connection network, diagnosability is an important problem that should be considered. In this article, a new concept regarding diagnosability, called strong local diagnosability (SLD), which describes the local status of the strong diagnosability (SD) of a system, is presented. In addition, a few important results related to the SLD of a node of a system are presented. Based on these results, we conclude that in a hypercube network of $n$ dimensions, denoted by $Q_{n}$, the SLD of a node is equal to its degree when $n \geqslant 4$. Moreover, we explore the SLD of a node of an incomplete hypercube network. We determine that the SLD of a node is equal to its remaining degree (RD) in an incomplete hypercube network, which is true provided that the number of faulty edges in this hypercube network does not exceed $(n-3)$. Finally, we discuss the SLD of a node for an incomplete hypercube network and obtain the following results: if the minimum RD of nodes in an incomplete hypercube network of $n$-dimensions is greater than 3, then the SLD of any node is still equal to its RD provided that the number of faulty edges does not exceed $7(n-3)-1$. If the $\mathrm{RD}$ of each node is greater than 4 , then the SLD of each node is also equal to its $\mathrm{RD}$, no matter how many faulty edges exist in $Q_{n}$.
\end{abstract}

\section{Introduction}

In a multiprocessor system incorporating a large number of processors (nodes), some processors may fail. In other words, there may exist faulty processors in such a system. Faulty processors in a multiprocessor system will affect the reliability of the system. Hence, in the system design, the problem of self-diagnosis should be considered. Whenever a faulty node is identified by the system, it should be repaired or replaced with an additional one. The process of identifying faulty processors is referred to as fault diagnosis, which has been widely studied in [1-9]. The diagnosability, a key measurement of self-diagnosis capacity, of a system is the upper limit on the number of faulty processors that a system is certain to identify.

The hypercube network structure [10] is a popular topology in modeling a multiprocessor system. An $n$-dimensional hypercube network, denoted as $Q_{n}$, consists of $2^{n}$ nodes and $n 2^{n-1}$ edges. A binary $n$-bit string, e.g., $x_{1} x_{2}, \ldots, x_{n}$, can be employed to denote the address of a node $x$ in $Q_{n}$. If the address of node $u$ and the address of node $v$ are different in exactly one bit position, then they are connected by a link, and vice versa. For two nodes $u$ and $v$ in $Q_{n}$, if $x$ and $y$ are connected by a link and their addresses differ in the $i$ th position, then this edge is called the $i$ th-dimensional edge. $Q_{n}$ can be decomposed in two $(n-1)$-dimensional hypercube networks $Q_{n-1}^{0}$ and $Q_{n-1}^{1}$ along the ith-dimensional edges. Let $v_{0} \in V\left(Q_{n-1}^{0}\right)$. Then there must be another node $v_{1} \in V\left(Q_{n-1}^{1}\right)$ satisfying $\left(v_{0}, v_{1}\right) \in E\left(Q_{n}\right)$. We call this edge the crossing edge between $Q_{n-1}^{0}$ and $Q_{n-1}^{1}$.

In system-level fault diagnosis, two models that are typically used as fault diagnosis models are the PMC model [11] and the comparison model [12]. The fault diagnosis model in this paper is the PMC model. "PMC" stands for 
Preparata, Metze, and Chien, as this model was first proposed by Preparata et al. in [11]. In [11], Preparata et al. put forward the concept of a one-step $t$-diagnosable system and of a sequentially $t$-diagnosable system. Since then, results corresponding to the PMC model have been widely reported (see [9, 11, 13-19]). In the PMC model, when we consider the diagnosability of a system $S$ with missing edges, if all neighbors of some node are faulty, then it is impossible to judge whether or not the node is fault-free. In other words, in the PMC model, the diagnosability of a system $S$ with missing edges depends on the upper limit of the minimum degree of $S$. In practice, the probability that all neighbors of a node are synchronously faulty is very low. For example, consider an $n$-dimensional hypercube network $Q_{n}$; the number of node subsets such that its cardinality is $n$ is $C_{2^{n}}^{n}$. However, the number of node subsets such that its size is $n$ and it incorporates all neighbors of some node is at most $2^{n}$. The ratio $2^{n} / C_{2^{n}}^{n}$ implies that the probability that there exists some node such that its neighbors are all faulty is very low. For this reason, Lai et al. [14] proposed a concept called a strongly $t$ diagnosable system (STDS). Lai et al. [14] claimed that a STDS is a $(t+1)$-diagnosable system provided that the system does not have a node such that its neighbors are all faulty simultaneously, the probability of which is very low. In other words, a STDS is nearly a $(t+1)$-diagnosable system. In a certain sense, the $\mathrm{SD}$ of a system is more precise than its traditional diagnosability. Much research on SD has been carried out (see $[2-4,6,9,14]$ ).

It is worth mentioning that in analyzing the diagnosability of a system, if one considers only the global situation and ignores some local information, it is possible that the diagnosability obtained is not the largest. For example, let $S$ denote the integrating system produced by $Q_{r}$ and $Q_{s}$, where $s>>r$. The diagnosability of $S$ may be less than $r$. However, the system may diagnose all faulty processors even if their number is greater than $r$ and less than $s$. Thus, it is possible that if some local details of a system are ignored, then the amount of details obtained will be less than that in the practical case. In practice, the local status of a system is also an indicative of the entire system. Hence, when we consider the problem of the diagnosability of a system, it is necessary to study the local properties of the system. In 2007, Hsu and Tan [20] proposed a novel diagnosability, called local diagnosability, in order to study the problem of fault diagnosis of a system under the PMC model. Later, in 2009, Chiang and Tan [13] extended the results in [20] from the PMC model to the comparison model. Now, we are led to the following question: is it possible to present a concept that describes the local status with respect to the SD of a system? This article presents a concept called SLD, which describes nodes' local SD status of a system. A sufficient and necessary condition, which tests the SLD of a node and its relationship with the SD of a system, is discussed in this article. Based on this sufficient and necessary condition, we conclude that the SD of a system can be determined by computing the SLD of each node of the system. Then, it is obtained that the SLD of a node of $Q_{n}$ is $n$, where $n \geqslant 4$. Moreover, we discuss the SLD of a given node of an incomplete hypercube network [21].
The remainder of this paper is as follows: after introducing some necessary preliminaries and the terminology used in this paper, in Section 3, we present the concept of SLD and an important theorem for checking the SLD of a given node in a system. In Section 4 , the SLD of a node of $Q_{n}$ is studied. Finally, in Section 5, we discuss the conclusions of our paper.

\section{Preliminaries}

A system is usually described by a graph $H(V, E)$, where $E$ represents all communication edges between processors and $V$ represents the set of all processors. Throughout this paper, we will use the following terms interchangeably: graph, multiprocessor system and interconnection system. Moreover, we will use node, vertex, and processor interchangeably. The definition of a graph follows that given in [22]. For a node $u$ in $H(V, E)$, a node $v$ is said to be its neighbor if $(u, v) \in E$. Moreover, let $N_{H}(z)=\{y \mid(y, z) \in E\} \quad(N(z)$, in no confusion) represent the set of all neighbors of $z$. The degree of a node $z$ in $H$, denoted by $\operatorname{deg}_{H}(z)$ (deg $(z)$, no confusion), is the cardinality of $N(z)$, i.e., $\operatorname{deg}(z)=|N(z)|$. If the degree of each node in $H$ is $k$, then $H$ is said to be $k$-regular. For a disconnected graph $H$, a connected component of $H$ refers to a maximal connected subgraph of $H$. If a connected component does not have any edges, it is said to be trivial; otherwise, it is nontrivial. Given a node subset $X$, let $H-X$ represent a subgraph created by deleting $X$ from $H$. Similarly, given an edge subset $E^{\prime}$, let $H-E^{\prime}$ represent a graph obtained by removing $E^{\prime}$ from $H$. The connectivity of $H$, denoted by $k(H)$, is the cardinality of the minimum node set $X(X \subset V)$ such that $H-X$ is disconnected. If $k(H) \geqslant r$, then $H$ is said to be $r$-connected. For a graph $H(V, E)$, we use $V(H)(E(H)$, respectively) to denote the node set of $H$ (the edge set of $H$, respectively).

In the PMC model, if $(x, y) \in E$, then $x$ and $y$ can be used to test each other. We use the order pair $(x, y)$ to represent that $x$ tests $y$. In this situation, $x$ is the tester, and $y$ is the tested node. The PMC model assumes that if $y$ is diagnosed to be faulty (fault-free) by $x$, then the outcome of $x$ testing $y$ is $1(0)$, denoted by $\sigma(x, y)=1(\sigma(x, y)=0)$. We use $\sigma=\{\sigma(x, y) \mid(x, y) \in E\}$ to denote a syndrome. The collection of all faulty nodes in $H$ is called a faulty set. It is possible that any subset of $V$ is a faulty set. The fault diagnosis of a system refers to the process of identifying all faulty nodes in the system. In a system $H$, the cardinality of a maximum faulty node set that can be identified by $H$ is called its diagnosability, denoted as $t(H)$. Given a syndrome $\sigma$, a set $S \subseteq V(H)$ is called consistent with $\sigma$ if the following condition is true: if $\forall x \in V(H)-S$ and $(x, y) \in E$, then $\sigma(x, y)=1$ if and only if $y \in S$.

We use $\sigma(S)$ to denote the set of syndromes produced by faulty node set $S$. Two subsets $X, Y \subseteq V(H)$ are called distinguishable if $\sigma(X) \cap \sigma(Y)=\varnothing$. If $X$ and $Y$ are distinguishable, then $(X, Y)$ is said to be a distinguishable pair; otherwise, $(X, Y)$ is said to be an indistinguishable pair. Let $X \Delta Y=(X-Y) \cup(Y-X)$. In addition, we need some previous results concerning the $t$-diagnosable 


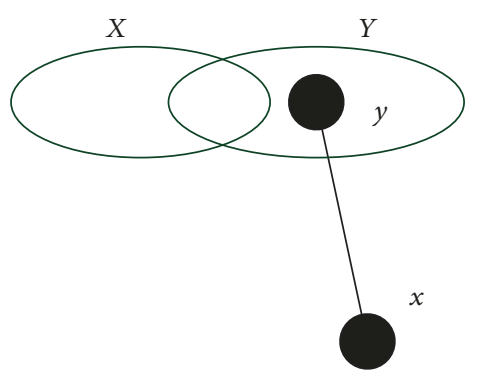

(a)

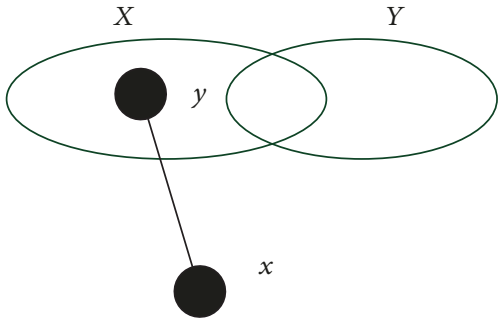

(b)

Figure 1: An example of a distinguishable pair $(X, Y)$.

system. Throughout this paper, the fault diagnosis model refers to the PMC model.

Lemma 1 (see [23]). A system $H(V, E)$ is t-diagnosable if and only if for two arbitrary different subsets $X, Y \subset V$ satisfying $|X| \leqslant t,|Y| \leqslant t,(X, Y)$ is always a distinguishable pair.

Lemma 2 (see [23]). In a system $H(V, E)$, suppose that $X, Y \subset V$ with $|X| \leqslant t,|Y| \leqslant t$ are two different subsets. Then $(X, Y)$ is a distinguishable pair if and only if $H$ has at least an edge $(x, y) \in E$ such that $x \in V-X-Y$ and $y \in X \Delta Y$ (Figure 1).

The following lemmas are related to the concept of node diagnosability:

Lemma 3 (see [20]). Suppose that $H(V, E)$ is a system, $z \in V$. $H$ is locally $t$-diagnosable at $z$ if and only if for each subset $X \subset V$ with $|X|=l, l \in\{0,1, \cdots, t-1\}$, and $z \notin X$, the number of nodes in the connected component containing $z$ in $H-X$ is greater than $2(t-l)$.

Lemma 4 (see [20]). Suppose that $H(V, E)$ is a graph, $v \in V$ with $\operatorname{deg}(v)=k$. Then, the locally diagnosability $(L D)$ of node $v$ does not exceed $k$.

Lemma 5 (see [20]). In a system $H(V, E)$, suppose that $z \in V$. Then the system $H(V, E)$ is $t$-diagnosable at node $z \in V(G)$ if and only if for two arbitrary different subsets $X, Y \subset V(H)$ satisfying $|X| \leqslant t,|Y| \leqslant t$ and $z \in X \Delta Y, H$ has at least an edge $(x, y) \in E$ such that $x \in V-X-Y$ and $y \in X \Delta Y$.

Lemma 6 (see [20]). A t-diagnosable system $H$ is locally $t$-diagnosable at each node. Conversely, if $H$ is locally $t$-diagnosable at each node, then $H$ is $t$-diagnosable.

By Lemma 1 and Lemma 5, we conclude that a $t$ diagnosable system must be $t$-diagnosable at each node, and vice versa.

Definition 1. A system is triangle-free if it does not incorporate a triangle.

Remark 1. Among the regular interconnection networks, there are many famous networks that are triangle-free, for example, hypercube-like networks [18], star networks [7], and the exchanged hypercube network [24].

The following definition of a strongly $t$-diagnosable system follows [14].

Definition 2. A $t$-diagnosable system, denoted by $H(V, E)$, is called a strong $t$-diagnosable system if the following condition is true.

For any two different subsets $X, Y \subset V$ with $|X| \leqslant t+1$ and $|Y| \leqslant t+1$, if $(X, Y)$ is an indistinguishable pair, then $H$ has at least a node $x$ such that $N(x) \subseteq X \cap Y$.

The strong diagnosability (SD) of a system is the maximum number value of $t$ such that the system is a strong $t$-diagnosable system.

\section{The Strong Local Diagnosability}

First, let us look back at some conclusions regarding diagnosability. Reference [20] proposed a new concept, called node diagnosability, that describes the local status with respect to system diagnosability. By Lemma 6, to obtain the system diagnosability, we need only to compute the node diagnosability of each node of the system, which is a novel way to study the system diagnosability. By Definition 2, we have that a STDS must be a $t$-diagnosable system but may not be a $(t+1)$-diagnosable system [13]. Since the probability that all neighbors of some node are faulty simultaneously is rather small, a STDS is almost $t+1$-diagnosable. Motivated by this strategy, we propose a new concept called the SLD at a given node in a system. This new concept combines the characteristics of node diagnosability and SD. For a system $H(V, E), x \in V$, we use $t(H)\left(t_{l} H(x) ; t_{s l} H(x)\right)$ to denote the diagnosability of $H(V, E)$ (the $\mathrm{LD}$ at node $x$ in $H(V, E)$; the SLD at node $x$ in $H(V, E)$ ).

Definition 3. Let $H(V, E)$ be the diagnostic graph of a system $H$ and $x \in V . H$ is called strong locally $t$-diagnosable at node $x$ if it is locally $t$-diagnosable at node $x$ and the following condition is true.

For any two different subsets $X, Y \subset V$ with $|X| \leqslant$ $t+1,|Y| \leqslant t+1$ and $x \in X \Delta Y$, if $(X, Y)$ is an indistinguishable pair, then $N(x) \subseteq X \cap Y$.

A system $H$ is said to be strong locally $t$-diagnosable if for each node $x$ in $H, H$ is strong locally $t$-diagnosable at node $x$. 
Proposition 1. Suppose that $H(V, E)$ is a graph, $y \in V$ with $\operatorname{deg}(y)=k$. The SLD of node $y$ in $H(V, E)$ does not exceed $k$.

Proof 1. By Lemma 4 and Definition 3, the proof can be easily obtained.

In the following, we propose two propositions for describing the relationship between a strong locally $t$ diagnosable system and a strongly $t$-diagnosable system:

Proposition 2. If $H(V, E)$ is strong locally $t$-diagnosable, then $H(V, E)$ is strongly t-diagnosable.

Proof 2. Suppose that $H(V, E)$ is not strongly $t$-diagnosable. From Definition 1, it is obtained that there is an indistinguishable pair $(X, Y)$ with $X, Y \subset V, X \neq Y,|X| \leqslant t+1$, and $|Y| \leqslant t+1$ such that for any node $x \in V, N(x) \not \subset X \cap Y$. Since $X \neq Y$, there exists a node $y$ such that $y \in X \Delta Y$ and $N(y) \not X \cap Y$. Hence, $H$ is not strong locally $t$-diagnosable at node $y$, a contradiction to the postulation that $H$ is strong locally $t$-diagnosable. This completes the proof.

The above proposition proposes a sufficient but unnecessary condition to test if the system $S$ is strongly $t$-diagnosable. Next, a necessary and sufficient condition for testing the strong $t$-diagnosability of a triangle-free system is presented.

Proposition 3. Let $H(V, E)$ be a triangle-free system and $t \geqslant 3$ be an integer. If $H$ is strongly $t$-diagnosable, then $H$ is strong locally t-diagnosable, and vice versa.

Proof 3. By the proof of Proposition 2, the sufficiency holds.

Necessity 1. Let $H$ be a strongly $t$-diagnosable system; then, $H$ is $t$-diagnosable. Let $x \in V$. By Lemma 6 , we have that $H$ is locally $t$-diagnosable at vertex $x$. Next, by Definition 3 , we need to prove only that for any two different subsets $X, Y \subset V$ with $|X| \leqslant t+1$ and $|Y| \leqslant t+1$, if $(X, Y)$ is an indistinguishable pair, then $H$ has at least a node $x \in X \Delta Y$ and $N(x) \subseteq X \cap Y$. In contrast, assuming that $N(x) \nsubseteq X \cap Y$, we derive a contradiction. By Definition 2, there is at least one node $y \in V$ such that $N(y) \subseteq N(X) \cap N(Y)$. By Lemma 4, we have that $|X \cap Y| \geqslant|N(y)| \geqslant t$. Note that $X \neq Y,|X| \leqslant$ $t+1$, and $|Y| \leqslant t+1$; therefore, $|X \cap Y| \leqslant t$. Thus, $|X \cap Y|=$ $|N(y)|=t$. On the other hand, $x \in X \Delta Y$ implies that $x \in X-Y$ or $x \in Y-X$. Here, we need to consider only the situation where $x \in X-Y$, as the proof of the situation in which $x \in Y-X$ can be similarly obtained. In the rest of this proof, four situations are considered according to the subjections of node $y$ : (1) $y \in X-Y$, (2) $y \in Y-X$, (3) $y \in X \cap Y$, and (4) $y \in V-(X \cup Y)$.

Case $1(y \in X-Y)$. Since $|X-Y| \leqslant 1$ and $X \neq Y, x=y$, which is a contradiction.

Case $2(y \in Y-X)$. It is clear that $Y-X=\{y\}, X-Y=\{x\}$. $(\mathrm{X}, Y)$ is an indistinguishable pair, implying that $N(x) \cap$ $(V-X \cup Y)=\phi$. Moreover, by $N(x) \nsubseteq X \cap Y$, we have that $y \in N(x)$. This is a contradiction to $N(y) \subseteq X \cap Y$.
Case $3(y \in X \cap Y)$. Since $|X \cap Y|=|N(y)|=t$ and $N(y) \subseteq$ $X \cap Y, N(y)=X \cap Y$, which is a contradiction.

Case $4(y \in V-(X \cup Y))$. By the hypothesis, we have that $X-Y=\{x\}$ and $|Y-X| \leqslant 1$. We first discuss the situation where $|Y-X|=1$. Suppose that $Y-X=\{z\}$. By the proof of Case 2, we have that $x$ is adjacent to $z$ and that there is no edge between $z$ and any node in $V-(X \cup Y) . N(x) \cap$ $(V-X \cap Y)=\phi \quad$ (as $(X, Y)$ is an indistinguishable pair) and $|N(x)| \geqslant t$ (Lemma 4), we have that $|N(x) \cup(X \cup Y)| \geqslant$ $t-1$. Since $H$ is a triangle-free graph, $|N(z) \cap(X \cap Y)| \leqslant 1$. Thus, the degree of $z$ is no greater than 2, a contradiction to $\operatorname{deg}(z) \geqslant t \geqslant 3$ (Lemma 4). Second, we consider the situation where $|Y-X|=0$. Since there is no edge between $x$ and any node in $V-(X \cup Y), N(x) \subseteq X \cup Y$; thus, $N(x) \subseteq X$, and therefore, $N(x) \cup\{x\} \subset X$. On the other hand, since $N(x) \geqslant t, X=\{x\} \cup N(x)$, and hence, $Y=N(x)$, which is a contradiction. This completes the proof.

Proposition 2 describes the relationship between SLD and SD for a triangle-free graph. The following theorem follows Proposition 2.

Theorem 1. In a triangle-free network system $H(V, E)$, the strong diagnosability of $H$ is equal to $\min$ \{the SLD at node $r \mid r \in V\}$.

The following sufficient and necessary condition can be applied.

Theorem 2. In a graph $H(V, E), x \in V$. Thus, $H(V, E)$ is strong locally t-diagnosable at node $x$ if and only if, for any $S \subset V,|S|=l, l \in\{0,1, \cdots, t\}$, and $x \notin S$, the following conditions hold:

Condition 1 If $l \neq t$, then the number of nodes of the connected component containing $x$ in $H-S$ exceeds $2(t+1-l)$.

Condition 2 If $l=t$, then either (a) the number of nodes of the connected component containing $x$ in $H-S$ exceeds 2 or $(b) N(x) \subseteq S$.

Proof 4 (sufficiency). Let $C$ denote the connected component containing $x$ in $H-S$. By Condition 1 , for $l \neq t$, we have that $2(t-l)+1<2(t+1-l)+1 \leqslant|V(C)|$. According to Lemma $3, H$ is locally $t$-diagnosable at node $x$. Next, we need to prove that for any two different subsets $X, Y \subset V$ with $|X| \leqslant t+1$, $|Y| \leqslant t+1$ and $x \in X \Delta Y$, if $(X, Y)$ is an indistinguishable pair, then $N(x) \subseteq X \cap Y$. Suppose that there exist two different subsets $X, Y \subset V$ with $|X| \leqslant t+1,|Y| \leqslant t+1$ and $x \in X \Delta Y$ such that $(X, Y)$ is an indistinguishable pair and $N(x) \nsubseteq$ $X \cap Y$. Let $S^{\prime}=X \cap Y$; then, $\left|S^{\prime}\right|=l \leqslant t$. If $l \neq t$, by Condition 1 , the number of nodes of the connected component containing $x$ in $H-S^{\prime}$ is greater than $2(t+1-l)$. Moreover, the number of nodes of the connected component containing $x$ in $H-S^{\prime}$ is greater than $|X \Delta Y|$, which implies that $H$ has two nodes $y \in V-X \cup Y$ and $z \in X \Delta Y$ such that $(y, z) \in E$. 
Hence, $(X, Y)$ is a distinguishable pair, which is a contradiction. Thus, $\left|S^{\prime}\right|=l=t$, which implies that $|X \Delta Y| \leqslant 2$. By Condition 2, we have that the number of nodes of the connected component containing $x$ in $H-S^{\prime}$ exceeds 2 . Hence, $H$ has two nodes $y \in V-X \cup Y$ and $z \in X \Delta Y$ such that $(y, z) \in E$. Hence, $(X, Y)$ is a distinguishable pair, which is a contradiction.

Next, we consider the situation $l=t$. From last paragraph, we have that $H$ is locally $t$-diagnosable at node $x$. By Definition 3, we only need to prove that for any two different subsets $X, Y \subset V$ with $|X| \leqslant t+1,|Y| \leqslant t+1$ and $x \in X \Delta Y$, if $(X, Y)$ is an indistinguishable pair, then $N(x) \subseteq X \cap Y$. To the contrary, assume that there exist two different subsets $X$, $Y \subset V$ with $|X| \leqslant t+1,|Y| \leqslant t+1, x \in X \Delta Y$ and $(X, Y)$ is an indistinguishable pair, but $N(x) \nsubseteq X \cap Y$. Let $S^{\prime}=X \cap Y$ and $\left|S^{\prime}\right|=r$, then $0 \leqslant r \leqslant t$, and then we have that $|X \Delta Y| \leqslant$ $2(t+1-r)$. Moreover, by Condition 1 , we have that $r=t$, which implies that $|X \Delta Y| \leqslant 2$. Since $(X, Y)$ is an indistinguishable pair, there is no edge from $V-(X \cup Y)$ to $X \Delta Y$. So, the number of nodes of the connected component containing $x$ is no more than $|X \Delta Y| \leqslant 2$. Moreover, $N(x) \subseteq S^{\prime}=$ $X \cap Y$, a contradiction.

Necessity 2. We consider Condition 1 first. Suppose that for some $l \in\{0,1, \cdots, t-1\}, H-S$ has a connected component containing $x$, e.g., $C$, such that $|V(C)| \leqslant 2(t+1-1)$. Let $V(C)$ be decomposed in two subsets as follows: $R_{1} \cap$ $R_{2}=\varnothing$ and $R_{1} \cup R_{2}=V(C)$, with $\left|R_{1}\right| \leqslant(t+1-l)$ and $\left|R_{2}\right| \leqslant$ $(t+1-l)$. Let $X=R_{1} \cup S$ and $Y=R_{2} \cup S$. It is clear that $|X| \leqslant$ $t+1,|Y| \leqslant t+1$ and $x \in X \Delta Y$. Since $C$ is a connected component of $H-S, H$ does not have an edge $(y, z) \in E$ such that $y \in X \Delta Y$ and $z \in V-(X \cup Y)$. By Lemma $2,(X, Y)$ is an indistinguishable pair. Since $H$ is strong locally $t$-diagnosable at node $x, N(x) \subseteq S$. Hence, $|N(x)| \leqslant|S| \leqslant l \leqslant t-1$. On the other hand, the assumption that $H$ is strong locally $t$ diagnosable at node $x$ implies that $H$ is $t$-diagnosable at node $x$. By Lemma 4 , we have that $|N(x)| \geqslant t$, which is a contradiction. Hence, Condition 1 is necessary.

Now, let us consider Condition 2. Suppose that $|S|=l=t$. Let $L$ denote the connected component containing $x$ in $H-S$. If the number of nodes in $L$ is less than or equal to 2 , then $V(L)$ can be decomposed in two subsets $R_{1}$ and $R_{2}$ satisfying the following conditions: $R_{1} \cap R_{2}=\varnothing$ and $R_{1} \cup R_{2}=V(L)$, with $\left|R_{1}\right| \leqslant 1$ and $\left|R_{2}\right| \leqslant 1$. Let $X=R_{1} \cup S$ and $Y=R_{2} \cup S$. Then, $|X| \leqslant t+1,|Y| \leqslant t+1$, and $x \in X \Delta Y$. By the assumption that $H(V, E)$ is strong locally $t$-diagnosable at node $x$, we have that $H(V, E)$ is locally $t$-diagnosable at node $x$. Moreover, we have that $(X, Y)$ is an indistinguishable pair. By Definition 3, we conclude that $N(x) \subseteq X \cap Y=S$. This completes the proof of necessity.

Corollary 1. Suppose that $H^{\prime}\left(V^{\prime}, E^{\prime}\right)$ is a subgraph of $H(V, E), y \in V^{\prime}$. If $H^{\prime}\left(V^{\prime}, E^{\prime}\right)$ is strong locally t-diagnosable at $y$ and $N_{H}(y) \subseteq V^{\prime}$, then $H(V, E)$ is strong locally t-diagnosable at $y$.

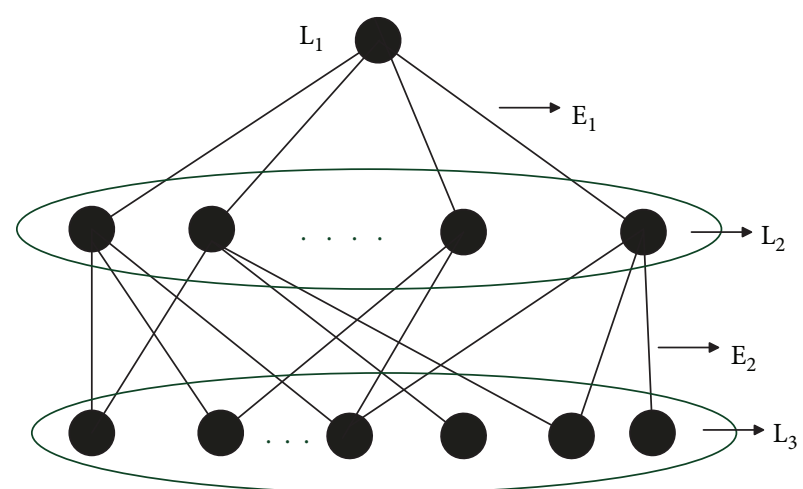

Figure 2: Type I structure.

Proof 5. For any $S \subset V,|S|=l, l \in\{0,1, \cdots, t\}$, and $x \notin S$. We will prove that the two conditions in Theorem 2 hold. Let $S^{\prime}=S \cap V^{\prime}, l^{\prime}=\left|S^{\prime}\right|$. Consider the following cases:

Case $5(l \neq t)$. Note that $l^{\prime} \neq t$. Since $H^{\prime}\left(V^{\prime}, E^{\prime}\right)$ is strong locally $t$-diagnosable at $y$, the number of nodes of the connected component containing $y$ in $H^{\prime}-S^{\prime}$ exceeds $2\left(t+1-l^{\prime}\right)$, which implies the number of nodes of the connected component containing $y$ in $H-S$ exceeds $2\left(t+1-l^{\prime}\right)$. Moreover, the number of nodes of the connected component containing $y$ in $H-S$ exceeds $2(t+1-l)$. So, Condition 1 of Theorem 2 holds.

Case $6(l=t)$.

Case $7\left(l^{\prime} \neq t\right)$. An argument being similar to Case 5 can be used to obtain the following result: the number of nodes of the connected component containing $y$ in $H^{\prime}-S^{\prime}$ exceeds 2 $\left(t+1-l^{\prime}\right)$, which implies that the number of nodes of the connected component containing $y$ in $H-S$ exceeds 2 . So, Condition 2 of Theorem 2 holds.

Case $8\left(l^{\prime}=t\right)$. Then $S^{\prime}=S$. Since $H^{\prime}\left(V^{\prime}, E^{\prime}\right)$ is strong locally $t$-diagnosable at $y$ and $N_{H}(y) \subseteq V^{\prime}$, then either (a) the number of nodes of the connected component containing $y$ in $H^{\prime}-S^{\prime}$ exceeds 2, which implies the number of nodes of the connected component containing $y$ in $H-S$ exceeds 2, or (b) $N_{H^{\prime}}(y) \subseteq S^{\prime}$, which implies $N_{H}(y) \subseteq S$. So, Condition 2 of Theorem 2 holds.

Next, we present the type I structure, which is used to verify the SLD of a given node.

Definition 4. Suppose that $H(V, E)$ denotes a diagnostic graph of a system and that $x$ is a node in $V$. The type I structure of the node $x$ can be decomposed in three node sets $\left(L_{1}, L_{2}\right.$, and $\left.L_{3}\right)$ and two edge sets $\left(E_{1}, E_{2}\right)$, as shown in Figure 2. These sets are defined as follows: $L_{1}=\{x\}, L_{2}=$ $\{y \mid(y, x) \in E\}$, and $L_{3}=\left\{z \mid(z, y) \in E, y \in L_{2}\right\} . E_{1}=\{(x, y)$ $\left.\mid x \in L_{1}, y \in L_{2}\right\}$, and $E_{2}=\left\{(y, z) \mid y \in L_{2}, z \in L_{3}\right\}$. Let $N_{n}$ $\left(N_{e}\right)$ represent the number of nodes (edges) in the type I structure. 


\section{The Hypercube Network and Incomplete Hypercube}

Regular topology structures are usually used to imitate multiprocessor systems. There is no doubt that the hypercube structure is one of the most important regular topology structures. In the following, we will discuss the problem of the SLD of a hypercube network.

Theorem 3. $Q_{n}$ is strong locally n-diagnosable at each node, where $n \geqslant 4$.

Proof 6. Let $v \in V\left(Q_{n}\right)$ be an arbitrary node. For each $S \subset V$ $\left(Q_{n}\right),|S|=l, l \in\{0,1, \cdots, n\}$, and $v \notin S$, we show that the two conditions of Theorem 2 are both true.

Case $9(l \neq n)$. Noting that the connectivity of $Q_{n}$ is $n$, we have that $Q_{n}-S$ is connected. Let $C$ denote this unique connected component in $Q_{n}-S$; then, $v \in V(C)$. Hence, $|V(C)|=2^{n}-l \geqslant 2(t+1-l)+1 \quad(n \geqslant 4)$, which implies that Condition 1 of Theorem 2 holds.

Case $10(l=n)$. Consider the type I structure of node $v$ in $Q_{n}$. If $S \subseteq L_{2}$, then $N(v) \subseteq S$, which implies that Condition 2 of Theorem 2 is true. If $S \nsubseteq L_{2}$, then there are two nodes $u, w$ satisfying $u \in L_{2}, w \in L_{3}$, and $(v, u) \in E_{1},(u, w) \in E_{2}$. Hence, $Q_{n}-S$ has a connected component, which incorporates $v$ and has 3 or more nodes. This completes the proof of Theorem 3.

According to Theorem 3, the SLD of every node of $Q_{n}$ is the same as its degree, with $n \geqslant 4$. It is natural to consider the following question: is the result still true for an incomplete hypercube network? In the following, we discuss this problem. We use $E^{\prime} \subset E$ to denote an edge subset and $Q_{n}^{\prime}$ to denote an incomplete hypercube network of $n$ dimensions created by removing $E^{\prime}$ from the hypercube network $Q_{n}$. In the following, we show that the SLD of every node is the same as its $\mathrm{RD}$ in $Q_{n}^{\prime}$, even if the cardinality of $E^{\prime}$ can reach $(n-3)$. We now give an example to explain that the result is not true if the cardinality of $E^{\prime}$ is $(n-2)$. In Figure 3, deg $(x)=n$. Let $E^{\prime}=\{(x, u) \in E \mid u \in V\}-\{(x, y)\}-\{(x, v)\}$, where $u$ and $v$ are two neighbors of $x$; then, $\left|E^{\prime}\right|=n-2$. Let $X=\left(N_{Q_{n}^{\prime}}(v)-\right.$ $\{x\}) \cup\{v, y\}$ and $Y=N_{Q_{n}^{\prime}}(v) \cup\{y\}$; then, $|X|=|Y|=n+1$, $v \in X \Delta Y$. By Lemma $2,(X, Y)$ is an indistinguishable pair, and $N(v) \subset X \cap Y$. Hence, the SLD of node $v$ differs from its $\mathrm{RD}$ in $Q_{n}^{\prime}$.

Theorem 4. Let $E^{\prime} \subset E\left(Q_{n}\right)$ be an edge subset with $0 \leqslant\left|E^{\prime}\right| \leqslant$ $n-3$, where $n \geqslant 4$. Let $Q_{n}^{\prime}$ denote the induced subgraph of $E\left(Q_{n}\right)-E^{\prime}$. Then, the SLD of every node of $Q_{n}^{\prime}$ is exactly the same as its $R D$.

Proof 7. We prove this theorem by induction $n$. First, let us consider the situation where $n=4$; in this case, $\left|E^{\prime}\right| \leqslant 1$. For $\left|E^{\prime}\right|=0$, based on Theorem 3, the result holds. Assume that

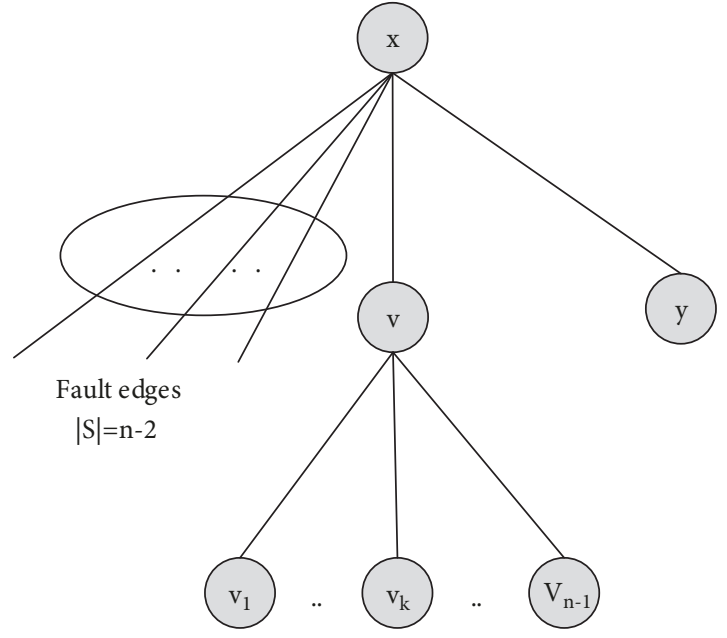

Figure 3

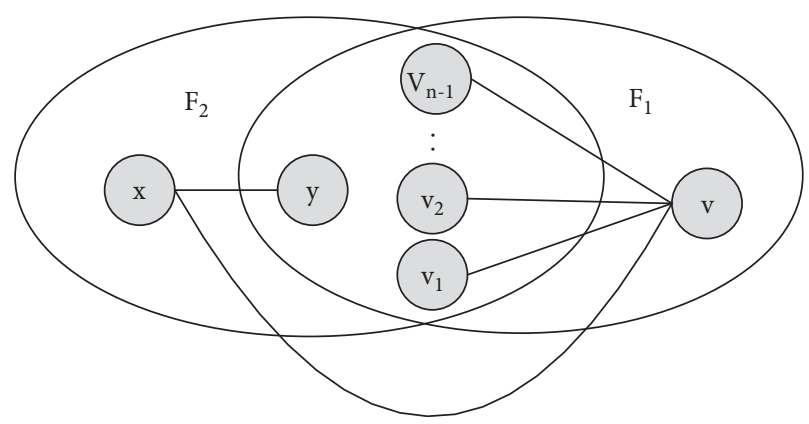

FIgURE 4: An illustration of the difference between the strong locally diagnosability and RD for a node in $Q_{n}^{\prime}$.

$\left|E^{\prime}\right|=1$. We consider the type I structure of node $v$ in $Q_{4}$, as shown in Figure 4. It is clear that $\left|L_{1}\right|+\left|L_{2}\right|+\left|L_{3}\right|=1+4+$ $6=11$. If $E^{\prime} \subset E_{1}$, then the RD of $v$ is 3 . We can use a similar argument to that used in the proof of Theorem 3 to show that the SLD of node $v$ is 3. If $E^{\prime} \subset E_{2}$, then the RD of $v$ is 4 . Let $S \subset V\left(Q_{4}^{\prime}\right)$ and $v \in S$, with $|S|=l$ and $0 \leqslant l \leqslant 4$. When $l=4$ and $N(v) \subseteq S$, Condition 2 of Theorem 2 holds. When $l=4$ and $N(v) \subseteq S$, consider the following cases.

Case $11(|N(v)-S| \geqslant 2)$. Then the number of nodes of the connected component containing $v$ in $Q_{4}^{\prime}-S$ is at least 3, Condition 2 of Theorem 2 holds.

Case $12(|N(v)-S|=1)$. Then $|N(v) \cap S|=3$. Let $u \in N(v)$ $-S$, then $N(u) \cap L_{3}$ has at least 2 nodes, which implies $\left|N(u) \cap L_{3}-S\right| \geqslant 1$. So, the number of nodes of connected component containing $v$ in $Q_{4}^{\prime}-S$ is at least 3. In other words, Condition 2 of Theorem 2 holds.

Next, we show that when $0 \leqslant l \leqslant 3,|V(C)| \geqslant 2(4+1-l)$ $+1=11-2 l$, where $C$ is the connected component containing $v$ in $Q_{4}^{\prime}-S$. From Figure 5, we conclude that the only case stopping the condition from being satisfied is that in which $S=\left\{v_{2}, v_{3}, v_{4}\right\}$ and $E^{\prime}=\left\{\left(v_{1}, v_{11}\right)\right\}$. However, based on the 


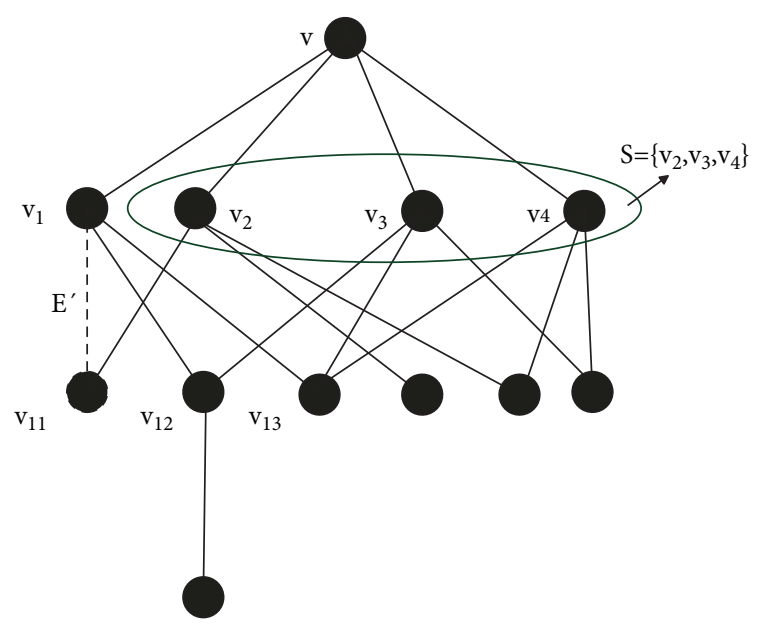

FIgURe 5: Type I structure of the node $v$ in $Q_{4}$.

definition of a hypercube network, the connected component $C$ contains other neighbors of $v_{1}$, e.g., $v_{12}$ and $v_{13}$, as well as their neighbors, which do not include $v_{1}$. Hence, for connected component $C$, it is true that $|V(C)| \geqslant 5$ when $l=3$. In summary, for $n=4$, the result is true.

Assume that when $n \geqslant 5$ for $Q_{n-1}^{\prime}$, the result is true. Next, we prove that for $n \geqslant 5, Q_{n}^{\prime}$ and $0 \leqslant\left|E^{\prime}\right| \leqslant(n-3)$, the result is still true. If $\left|E^{\prime}\right|=0$, then the result holds. Now, we consider the situation where $1 \leqslant\left|E^{\prime}\right| \leqslant n-3$. Decompose $Q_{n}$ in two$(n-1)$ dimensional hypercube networks $Q_{n-1}^{0}$ and $Q_{n-1}^{1}$ along the $i$ th-dimensional edges. Let $E_{0}^{\prime}=E\left(Q_{n-1}^{0}\right) \cap E^{\prime}, E_{1}^{\prime}=$ $E\left(Q_{n-1}^{1}\right) \cap E^{\prime}$. Let $v$ be an arbitrary node. Without the loss of generality, let $v \in V\left(Q_{n-1}^{0}\right)$ and $\operatorname{deg}_{Q_{n-1}^{0}-E_{0}^{\prime}}(v)=k$. Suppose that $\left(v, v^{\prime}\right) \in E\left(Q_{n}\right)$ is a crossing edge, namely, $\left(v, v^{\prime}\right) \in$ $E\left(Q_{n}\right)$ is one of the ith-dimensional edges, where $v^{\prime} \in$ $V\left(Q_{n-1}^{1}\right)$. Consider the following two cases:

Case $13\left(\left(v, v^{\prime}\right) \in E^{\prime}\right)$. Then, $0 \leqslant\left|E_{0}^{\prime}\right| \leqslant(n-1)-3$. By the inductive hypothesis, we have that $t_{s l}\left(Q_{n-1}^{0}-E_{0}^{\prime}\right)(v)=k$. On the other hand, we have that $\operatorname{deg}_{Q_{n}-E^{\prime}}(v)=k$. According to Corollary 1 and Proposition 1 , we have that $k=t_{s l}\left(Q_{n}^{0}-E_{0}^{\prime}\right)$ $(v) \leqslant t_{s l}\left(Q_{n}-E^{\prime}\right)(v) \leqslant \operatorname{deg}_{Q_{n}-E^{\prime}}(v)=k$. Hence, $t_{s l}\left(Q_{n}-E^{\prime}\right)$ $(v)=k$, and thus the result is true.

Case $14\left(\left(v, v^{\prime}\right) \notin E^{\prime}\right)$. Then, $\operatorname{deg}_{Q_{n}-E^{\prime}}(v)=k+1$. By Proposition 1 , we have that $t_{s l}\left(Q_{n}^{\prime}-E^{\prime}\right)(v) \leqslant k+1$. Now, we need to prove only that $Q_{n}-E$ is strong locally $k+1$-diagnosable at node $v$. Let $S \subset V\left(Q_{n}-E^{\prime}\right)$, with $v \notin S, 0 \leqslant|S|=l \leqslant k+1$, $S_{0}=S \cap V\left(Q_{n-1}^{0}-E_{0}^{\prime}\right)$, and $S_{1}=S \cap V\left(Q_{n-1}^{1}-E_{1}^{\prime}\right)$. Next, we prove that the two conditions of Theorem 2 hold. For the sake of convenience, let $C$ stand for the connected component containing $v$ in $\left(Q_{n}-E^{\prime}\right)-S, C_{0}$ denote the connected component containing $v$ in $\left(Q_{n-1}^{0}-E_{0}^{\prime}\right)-S_{0}$, and $C_{1}$ denote the connected component containing $v^{\prime}$ in $\left(Q_{n-1}^{1}-E_{1}^{\prime}\right)-S_{1}$; moreover, let $s_{i}=\left|S_{i}\right|, i=0,1$.
Case 15. First, we consider the condition that $l \leqslant k$. We will prove that $|V(C)| \geqslant 2(k+1+1-l)+1$. Consider the following cases:

Case $16\left(\left|E_{0}^{\prime}\right|=(n-3)\right)$.

Case $17\left(v^{\prime} \notin S\right)$. If $s_{1} \leqslant n-2$, then $\left|V\left(C_{1}\right)\right| \geqslant 2^{n-1}-s_{1}$. Moreover, $|V(C)| \geqslant\left|V\left(C_{1}\right) \cup\{v\}\right| \geqslant 2^{n-1}+1-l \geqslant 2(k+1+$ $1-l)+1$. If $s_{1}=n-1$, then $\left|V\left(C_{0}\right)\right|=2^{n-1}$. Moreover, $|V(C)| \geqslant\left|V\left(C_{0}\right) \cup\left\{v^{\prime}\right\}\right| \geqslant 2^{n-1}+1 \geqslant 2(k+1+1-l)+1$.

Case $18\left(v^{\prime} \in S\right)$. It is obvious that $s_{0} \leqslant k-1$. Let $(x, y) \in E_{0}^{\prime}$; then, $\{x, y\}$ has at least one node, e.g., $x$, which is not $v$. Let $\bar{S}_{0}=S_{0} \cup\{x\}$; then, $\left|\bar{S}_{0}\right| \leqslant s_{0}+1 \leqslant k$.

If $\left|\bar{S}_{0}\right| \leqslant k-1$, let $\bar{C}_{0}$ denote the connected component containing $v$ in $\left(Q_{n-1}^{0}-\left(E_{0}^{\prime}-\{(x, y)\}\right)-\bar{S}_{0}\right)$. Since $Q_{n-1}^{0}-$ $\left(E_{0}^{\prime}-\{(x, y)\}\right)-\bar{S}_{0}$ is the subgraph of $Q_{n-1}^{0}-E_{0}^{\prime}-S_{0}$, $\left|V\left(C_{0}\right)\right| \geqslant\left|V\left(\bar{C}_{0}\right)\right| . \quad$ Note that $\left|E_{0}^{\prime}-\{(x, y)\}\right|=(n-1)-3$; by inductive hypothesis and Theorem 2 , we have that $\left|V\left(\bar{C}_{0}\right)\right| \geqslant 2\left(k+1-\left(s_{0}+1\right)\right)+1$. In $Q_{n-1}^{0}-E_{0}^{\prime}-S_{0}$, we can choose two distinct nodes $u$ and $w$ such that either both $u$ and $w$ are neighbors of $v$ or $u$ is a neighbor of $v$ and $w$ is a neighbor of $u$. Suppose that $\left(u, u^{\prime}\right)$ and $\left(w, w^{\prime}\right)$ are two crossing edges in $Q_{n}$, where $u^{\prime}, w^{\prime} \in V\left(Q_{n-1}\right)^{1}$ and $\left(u, u^{\prime}\right)$, $\left(w, w^{\prime}\right) \in E$. If $s_{1}=1$, then $V\left(\bar{C}_{0}\right) \cup\left\{u^{\prime}, w^{\prime}\right\} \subset V(C)$, and then $|V(C)| \geqslant\left|V\left(\bar{C}_{0}\right)\right|+2 \geqslant 2\left(k+1-\left(s_{0}+1\right)\right)+1+2=2((k+1)$ $+1-l)+1$. If $s_{1} 1$, then $s_{0}+1 \leqslant l-1$, and then $|V(C)| \geqslant$ $\left|V\left(\bar{C}_{0}\right)\right| \geqslant 2\left(k+1-\left(s_{0}+1\right)\right)+1 \geqslant 2((k+1)+1-l)+1$.

If $\left|\bar{S}_{0}\right|=k$, then $s_{1}=1, s_{0}=k-1$. Since $\operatorname{deg}_{Q_{n-1}^{0}-E_{0}^{\prime}}(v)=k$, there must exist one neighbor of $v$, e.g., $z$, such that $z \neq$ $x$ in $Q_{n-1}^{0}-\left(E_{0}^{\prime}-\{(x, y)\}\right)-\bar{S}_{0}$. Let $\left(z, z^{\prime}\right)$ be a crossing edge in $Q_{n}$, where $z^{\prime} \in V\left(Q_{n-1}^{1}\right)$. Let $C_{z^{\prime}}$ denote the connected component containing $z^{\prime}$ in $\left(Q_{n-1}^{1}-E_{1}^{\prime}\right)-S_{1}$; then, $\left|V\left(C_{z^{\prime}}\right)\right| \geqslant 2^{n-1}-s_{1}$. Note that $\left(z, z^{\prime}\right) \notin E^{\prime}$; we have that $v, z, z^{\prime}$ belong to a connected component of $Q_{n}-E^{\prime}$, which implies that $|V(C)| \geqslant\left|V\left(C_{z^{\prime}}\right) \cup\{v, z\}\right| \geqslant 2^{n-1}+2-$ $s_{1} \geqslant 2(k+1+1-l)+1$.

Case $19\left(\left|E_{0}^{\prime}\right|=0\right)$.

If $v^{\prime} \in S$, then $s_{0} \leqslant k-1 \leqslant n-2$. Since the connectivity of $Q_{n-1} \quad$ is $n-1, \quad\left|V\left(C_{0}\right)\right| \geqslant 2^{n-1}-s_{0} \geqslant 2(k+1+1-l)+1$. Hence, $|V(C)| \geqslant\left|V\left(C_{0}\right)\right| \geqslant 2(k+1+1-l)+1$. If $v^{\prime} \notin S$, then either $\left|V\left(C_{0}\right)\right| \geqslant 2^{n-1}-s_{0} \geqslant 2(k+1+1-l)+1$ or $\left|V\left(C_{1}\right)\right| \geqslant$ $2^{n-1}-s_{1} \geqslant 2(k+1+1-l)+1$. Hence, $|V(C)| \geqslant 2(k+1+$ $1-l)+1$.

Case $20\left(1 \leqslant\left|E_{0}^{\prime}\right| \leqslant(n-1)-3\right)$. Next, we show that $|V(C)|$ $\geqslant 2((k+1)+1-l)+1$. Since $0 \leqslant\left|E_{1}^{\prime}\right| \leqslant(n-3)-1$, the degree of any node in $Q_{n-1}^{1}-E_{1}^{\prime}$ is at least 3. Therefore, $v^{\prime}$ has two neighbors, e.g., $x$ and $y$, in $Q_{n-1}^{1}-E_{1}^{\prime}$. Consider the following cases: 


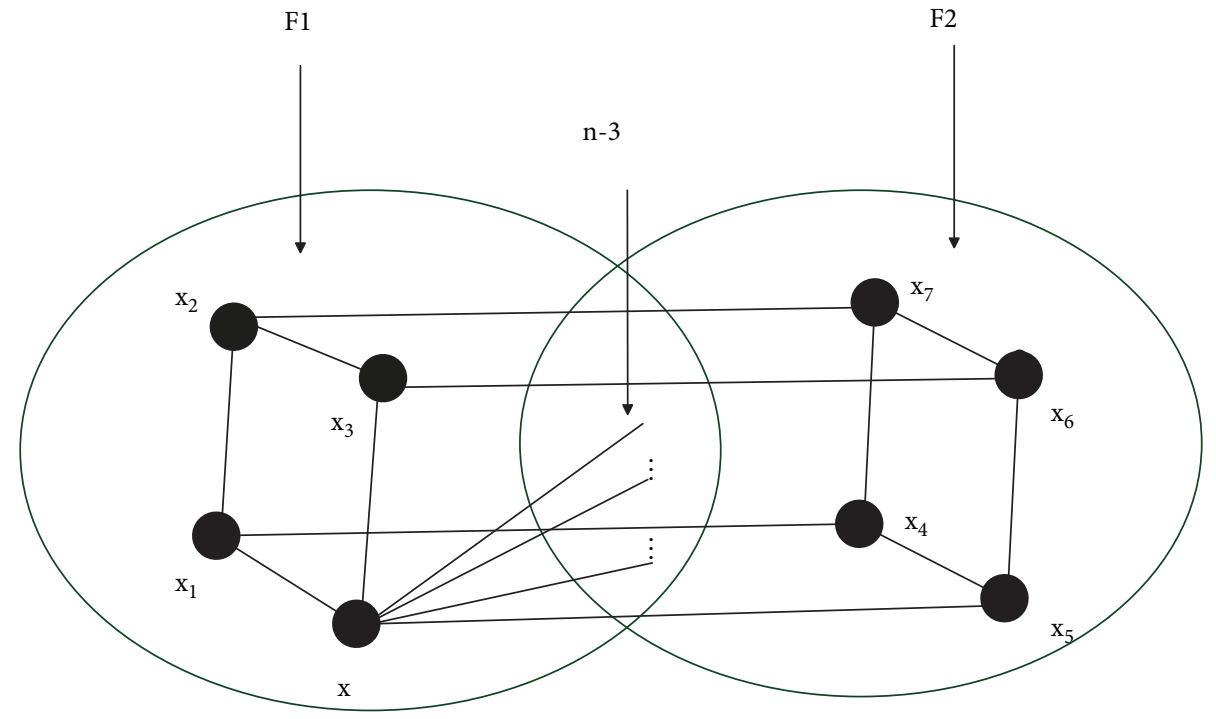

FIgURE 6: Illustration of $t_{s l}\left(Q_{n}-E^{\prime}\right)(x)$ being equal to its RD.

Case $21(0 \leqslant l \leqslant k-1)$. By inductive hypothesis and Theorem 2 , we have that $\left|V\left(C_{0}\right)\right| \geqslant 2\left(k+1-s_{0}\right)+1$. If $S \subset V\left(Q_{n-1}^{0}-\right.$ $\left.E_{0}^{\prime}\right)$, then $|V(C)| \geqslant\left|V\left(C_{0}\right)\right|+\left|\left\{v^{\prime}, x, y\right\}\right| \geqslant 2(k+1-l)+1+$ $3 \geqslant 2((k+1)+1-l)+1$. If $S \subseteq V\left(Q_{n-1}^{0}-E_{0}^{\prime}\right)$, then $|V(C)| \geqslant$ $\left|V\left(C_{0}\right)\right| \geqslant 2(k+1-(l-1))+1=2((k+1)+1-l)+1$.

Case $22(l=k)$. Since $0 \leqslant\left|E_{1}^{\prime}\right| \leqslant(n-3)-1$, there exists another neighbor of $v^{\prime}$, e.g., $v_{11}^{\prime}$, in $Q_{n-1}^{1}-E_{1}^{\prime}$. If $S \subseteq Q_{n-1}^{0}-$ $E_{0}^{\prime}$, then $\left|V\left(C_{1}\right)\right| \geqslant 2^{n-1}$, and then $|V(C)| \geqslant\left|V\left(C_{1}\right) \cup\{v\}\right| \geqslant$ $2^{n-1}+1 \geqslant 2((k+1)+1-l)+1$. If $S \subseteq Q_{n-1}^{0}-E^{\prime}$, then $s_{0} \leqslant k$ -1 . Hence, by inductive hypothesis and Theorem 2 , we have that $\left|V\left(C_{0}\right)\right| \geqslant 2\left(k+1-\left(s_{0}\right)\right)+1 \geqslant 2((k+1)-l)+1$.

Case 23. Next, we consider the condition that $l=k+1$. Suppose that $N_{Q_{n}^{\prime}}(v) \subseteq S$. Consider the following cases.

Case $24\left(v^{\prime} \notin S\right)$. Since $\left|N_{Q_{n-1}^{1}-E_{1}^{\prime}}\left(v^{\prime}\right)\right|+\left|N_{Q_{n-1}^{0}-E_{0}^{\prime}}(v)\right| \geqslant 2(n-$ $1)-(n-3)=n+1>k+1=|S|$, there is at least node $y$ in $N_{Q_{n-1}^{1}-E_{1}^{\prime}}\left(v^{\prime}\right) \cup N_{Q_{n-1}^{0}-E_{0}^{\prime}}(v)$ such that $y \notin S$. Hence, $y, v, v^{\prime}$ belong to a connected component in $Q_{n}-E^{\prime}-S$. Condition 2 of Theorem 2 is satisfied.

Case $25\left(v^{\prime} \in S\right)$. By $N_{Q_{n}-E^{\prime}}(v) \nsubseteq S$, we conclude that there exists at least node in $N_{Q_{n-1}^{0}-E_{0}^{\prime}}(v)$, e.g., $y$, such that $y \notin S$. Since $N_{Q_{n-1}-E^{\prime}}\{v, y\} \geqslant n+1>k+1=|S|$, then there is at least one node in $N_{Q_{n}-E^{\prime}}$, e.g., $z$, such that $z \notin S$. Then, $x, y, z$ belong to a connected component in $Q_{n}-E^{\prime}-S$. Condition 2 of Theorem 2 is satisfied.

In summary, $Q_{n}-E^{\prime}$ is strong locally $(k+1)$-diagnosable at the node $v$.

Clearly, the following corollary follows Theorem 4; hence, its proof is omitted.
Corollary 2. For $n \geqslant 4$ and $E^{\prime} \subset E\left(Q_{n}\right)$ with $0 \leqslant\left|E^{\prime}\right| \leqslant n-3$, $Q_{n}-E^{\prime}$ is strongly $r$-diagnosable, where $r$ represents the minimum degree of nodes in $Q_{n}-E^{\prime}$.

In the previous section, we concluded that the SLD of a node is its $\mathrm{RD}$ in an incomplete hypercube network $Q_{n}^{\prime}$ created by removing the edge set $E^{\prime} \subset E\left(Q_{n}\right)$ with $\left|E^{\prime}\right| \leqslant n-3$ from $Q_{n}$. In the following, we discuss the problem of the upper bound of $\left|E^{\prime}\right|$ such that the following two conditions are satisfied: (1) the $\mathrm{RD}$ of each node in $Q_{n}-E^{\prime}$, denoted by $Q_{n}^{\prime}$, is at least 4 ; (2) the SLD of every node $Q_{n}^{\prime}$ is the same as its RD. Now, we consider an example in which the SLD of a node in $Q_{n}^{\prime}$ differs from its $\mathrm{RD}$ in $Q_{n}^{\prime}$ with $\left|E^{\prime}\right|=7(n-3)$, even though the minimum degree of nodes in $Q_{n}^{\prime}$ is more than 3 . Assume that $x, x_{i} \in V, 1 \leqslant i \leqslant 7$, the adjacent situations of which are shown in Figure 6. Let $H\left(x_{i}\right)=N\left(x_{i}\right)-$ $\left\{x_{1}, x_{2}, \cdots, x_{7}\right\}, E_{i}=\left\{\left(y, x_{i}\right) \in E \mid y \in H\left(x_{i}\right)\right\}$, and $E^{\prime}=E_{1} \cup$ $E_{2} \cup \cdots \cup E_{7}$; then, $\left|E^{\prime}\right|=7(n-3)$. Let $F_{1}=\left(N(x)-\left\{x_{5}\right\}\right) \cup$ $\left\{x, x_{2}\right\}$ and $F_{2}=\left(N(x)-\left\{x_{1}, x_{3}\right\}\right) \cup\left\{x_{4}, x_{6}, x_{7}\right\}$. Since $\left|F_{1}\right|=\left|F_{2}\right|=n+1,\left(F_{1}, F_{2}\right)$ is an indistinguishable pair; at the same time, $N(x) \subseteq F_{1} \cap F_{2}, t_{s l}\left(Q_{n}-E^{\prime}\right)(v)$ is not $n$, and the $\mathrm{RD}$ of $v$ is $n$ in $Q_{n}-E^{\prime}$.

Theorem 5. Let $Q_{n}^{\prime}=Q_{n}-E^{\prime}$, where $E^{\prime} \subset E\left(Q_{n}\right)$ with $0 \leqslant\left|E^{\prime}\right| \leqslant 7(n-3)-1$. Assume that $\delta\left(Q_{n}^{\prime}\right) \geqslant 3$, then $\forall$ $x \in V\left(Q_{n}^{\prime}\right)$, it follows that $t_{s l}\left(Q_{n}{ }^{\prime}\right)(x)=\operatorname{deg}_{Q_{n}^{\prime}}(x)$.

Proof 8 . Before proving this theorem, we define some notions for further discussion. Consider another structure of a node $v$ obtained by extending its type I structure, called the type I-EX structure of node $v$, in the following way: insert a node set $L_{4}$ and an edge set $E_{3}$ in the type I structure of node $v$, where $L_{4}=\left\{u \mid(u, w) \in E, w \in L_{3}\right\}-L_{2}$ and $E_{3}=$ $\left\{(u, w) \mid u \in L_{3}, w \in L_{4}\right\}$. After removing $E^{\prime}$ from $Q_{n}$, the type I-EX structure of node $v$ becomes another new structure, 
called the type II structure of node $v$. The set of nodes $L_{1}$ $\left(L_{2} ; L_{3} ; L_{4}\right)$ becomes another new set of nodes $L_{1}^{\prime}\left(L_{2}^{\prime} ; L_{3}^{\prime} ; L_{4}^{\prime}\right)$ in the type II structure of node $v$. Similarly, the set of edges $E_{1}\left(E_{2} ; E_{3}\right)$ becomes another new set of edges $E_{1}^{\prime}$ $\left(E_{2}^{\prime} ; E_{3}^{\prime}\right)$ in the type II structure of node $v$. Let $\operatorname{deg}_{Q_{n}-E^{\prime}}(v)=h$ and $S \subset V\left(Q_{n}\right)$ with $|S|=l$ and $0 \leqslant l \leqslant h$. By the type II structure of node $v$, we have that the number of nodes of connected component containing $v$ in $Q_{n}^{\prime}-S$ is smallest if and only if $S \subset L_{2}^{\prime}$. So, we need to consider only the case of $S \subset L_{2}$.

If $|S|=h$, it is obtained that $N(v) \subseteq S$, which satisfies Condition 2 of Theorem 2. Now, we consider the case of $0 \leqslant|S| \leqslant h-1$. For the sake of convenience, we introduce another new structure of node $v$, called type III structure of node $v$, which is obtained by removing $S$ from the type II structure of node $v$. The set of nodes $L_{1}^{\prime}\left(L_{2}^{\prime} ; L_{3}^{\prime} ; L_{4}^{\prime}\right)$ becomes $L_{1}^{\prime \prime}\left(L_{2}^{\prime \prime} ; L_{3}^{\prime \prime} ; L_{4}^{\prime \prime}\right)$ in type III structure of node $v$. Similarly, the set of edges $E_{1}^{\prime}\left(E_{2}^{\prime}\right.$; $\left.E_{3}^{\prime}\right)$ becomes another new set of edges $E_{1}^{\prime \prime}\left(E_{2}^{\prime \prime} E_{3}^{\prime \prime}\right)$ in the type III structure of node $v$. After deleting all nodes of $S$, we have $\left|L_{1}^{\prime \prime}\right|=1$ and $\left|L_{2}^{\prime \prime}\right|=h-l$. Since for each $v \in V\left(Q_{n}^{\prime}\right), \operatorname{deg}_{Q_{n}^{\prime}}(v) \geqslant 3$, it follows that $\left|E_{1}^{\prime \prime}\right|=h-l$ and $\left|E_{2}^{\prime}\right| \geqslant 2(h-l)$. Moreover, by the definition of $Q_{n}$, we have that each node in $L_{3}^{\prime \prime}$ is adjacent to at most two nodes in $L_{2}^{\prime \prime}$ Thus, it is true that $\left|L_{3}^{\prime \prime}\right| \geqslant\left(\left|E_{2}^{\prime \prime}\right| / 2\right) \geqslant h-l$ and $\left|E_{3}^{\prime \prime}\right| \geqslant h-l$. Similarly, it is true that each node in $L_{4}^{\prime \prime}$ is adjacent to at most three nodes in $L_{3}^{\prime \prime}$; thus, $\left|L_{4}^{\prime}\right| \geqslant\left(\left|E_{3}^{\prime}\right| / 3\right) \geqslant\lceil h-l / 3\rceil$. Hence, for the component $C$, to which $v$ in $Q_{n}^{\prime}-S$ belongs, we have that $|V(C)| \geqslant 1+2(h-l)+\lceil h-l / 3\rceil$. To show that $2(h+1-l)+$ $1 \leqslant|V(C)|$, which implies that Condition 1 of Theorem 2 holds, we consider the following cases:

Case $26(h-l \geqslant 4)$. Since $\lceil h-l / 3\rceil \geqslant 2$, it follows that $|V(C)| \geqslant 1+2(h-l)+\lceil h-l / 3\rceil \geqslant 2(h+1-l)+1$.

Case $27(h-l=3)$. Note that $2(h+1-l)+1=9$ and $1+2$ $(h-l)+\lceil h-l / 3\rceil=8$; hence, it is sufficient that $V(C) \neq 8$. Suppose that $V(C)=8$. Since $h-l=3,\left|L_{1}^{\prime}\right|=1$, and $\left|L_{2}^{\prime}\right|=3$ in the type II structure of node $v$, the assumption that for each $v \in V\left(Q_{n}^{\prime}\right), \operatorname{deg}_{Q_{n}^{\prime}}(v) \geqslant 3$ implies that $\left|L_{3}^{\prime}\right|=4$ or $\left|L_{3}^{\prime}\right|=3$. If $\left|L_{3}^{\prime}\right|=4$, then there exists some node $v_{0} \in L_{3}^{\prime}$ such that there is exactly one node in $L_{3}^{\prime}$ that can connect to $v_{0}$. Then, $L_{4}^{\prime}$ has at least one node, e.g., $v_{0}^{\prime}$, such that $\left(v_{0}, v_{0}^{\prime}\right) \in E^{\prime}$. In other words, $V(C) \geqslant 9$, which contradicts the assumption that $V(C)=8$. If $\left|L_{3}^{\prime}\right|=3$, then $\forall u \in L_{3}^{\prime}$, it follows that $\operatorname{deg}_{Q_{n}^{\prime}}(u)=$ $3,\left|L_{4}^{\prime}\right|=1,\left|E_{3}^{\prime}\right|=3$, which implies that there exist 7 nodes in $Q_{n}^{\prime}$ whose $\mathrm{RDs}$ are all 3. This contradicts the assumption that $0 \leqslant\left|E^{\prime}\right| \leqslant 7(n-3)-1$.

Case $28(h-l=2)$. Note that $2(h+1-l)+1=7$ and $1+2$ $(h-l)+\lceil h-l / 3\rceil=6$; hence, it is sufficient that $V(C) \neq 6$. Suppose that $V(C)=6$. Since $h-l=2$, it follows that $\left|L_{1}^{\prime}\right|=$ 1 and $\left|L_{2}^{\prime}\right|=2$ in the type II structure of node $v$. On the other hand, the assumption that for each $v \in V\left(Q_{n}^{\prime}\right), \operatorname{deg}_{Q_{n}^{\prime}}(v) \geqslant 3$ implies that $\left|L_{3}^{\prime}\right|=3$, where there exists at most one node in $L_{3}^{\prime}$ that is shared by the two nodes in $L_{2}^{\prime}$ as their common neighbor. Furthermore, $\left|L_{4}^{\prime}\right| \geqslant 1$ can be obtained, which implies that $V(C) \geqslant 7$. This is a contradiction.

Case $29(h-l=1)$. Note that $2(h+1-l)+1=5$ and $1+2$ $(h-l)+\lceil h-l / 3\rceil=4$; hence, it is sufficient that $V(C) \neq 4$. Suppose that $V(C)=4$. Since $h-l=1$, it follows that $\left|L_{1}^{\prime}\right|=1$ and $\left|L_{2}^{\prime}\right|=1$ in the Type II structure of node $v$. The assumption that for each $v \in V\left(Q_{n}^{\prime}\right), \operatorname{deg}_{Q_{n}^{\prime}}(v) \geqslant 3$ implies that $\left|L_{3}^{\prime}\right| \geqslant 2$, which results in $\left|L_{4}^{\prime}\right| \geqslant 1$. Hence, $V(C) \geqslant 5$, which contradicts the assumption that $V(C)=4$.

By Theorem 5, we can obtain the following corollary.

Corollary 3. In $Q_{n}(n \geqslant 4)$, suppose that $E^{\prime} \subset E\left(Q_{n}\right)$, with $0 \leqslant\left|E^{\prime}\right| \leqslant 7(n-3)-1$. Then, $Q_{n}-E^{\prime}$ is strongly r-diagnosable, where $r$ represents the minimum degree of all nodes in $Q_{n}-E^{\prime}$ and $r \geqslant 3$.

Finally, we discuss the situation where the $\mathrm{RD}$ of each node in $Q_{n}-E^{\prime}$ exceeds 3.

Theorem 6. In $Q_{n}(n \geqslant 4)$, let $E^{\prime} \subset E\left(Q_{n}\right)$, where $Q_{n}^{\prime}=Q_{n}-E^{\prime}$. If for each $v \in V\left(Q_{n}^{\prime}\right), \operatorname{deg}_{Q_{n}^{\prime}}(v)>3$, then for any $x \in V\left(Q_{n}^{\prime}\right)$, it follows that $t_{s l}\left(Q_{n}{ }^{\prime}\right)(x)=\operatorname{deg}_{Q_{n}^{\prime}}(x)$.

Proof 9. We follow all definitions and terminologies mentioned in the proof of Theorem 5. Let $S \subset V\left(Q_{n}^{\prime}\right)$, with $|S|=$ $l$ and $0 \leqslant l \leqslant k$. As in the proof of Theorem 5 , we need to prove only that $|V(C)| \geqslant 2(k+1-l)+1$ for $0 \leqslant l \leqslant k-1$ and $S \subseteq L_{2}$. Since for each $v \in V\left(Q_{n}^{\prime}\right), \operatorname{deg}_{Q_{n}^{\prime}}(v)>3$, we have that $\left|L_{1}^{\prime}\right|=1,\left|L_{2}^{\prime}\right|=k-l$ and $\left|E_{1}^{\prime}\right|=k-l,\left|E_{2}^{\prime}\right| \geqslant 3(k-l)$. Therefore, we have that $\left|L_{3}^{\prime}\right| \geqslant\left(\left|E_{2}^{\prime}\right| / 2\right) \geqslant(3(k-l) / 2),\left|E_{3}^{\prime}\right| \geqslant(4-2)\left|L_{3}^{\prime}\right|$ $\geqslant 3(k-l)$ and $\left|L_{4}^{\prime}\right| \geqslant\left(\left|E_{3}^{\prime}\right| / 3\right) \geqslant k-l$. Thus, $|V(C)| \geqslant\left|L_{1}^{\prime}\right|+$ $\left|L_{2}^{\prime}\right|+\left|L_{3}^{\prime}\right|+\left|L_{4}^{\prime}\right| \geqslant 1+(k-l)+(3(k-l) / 2)+(k-l)=2(k-l)$ $+1+(3(k-l) / 2)$. Then, we need to prove that $\lceil 3(k-l) / 2\rceil$ $\geqslant 2$. Clearly, the result holds when $0 \leqslant l \leqslant k-1$. Hence, for any $x \in V\left(Q_{n}^{\prime}\right), t_{s l}\left(Q_{n}{ }^{\prime}\right)(x)=\operatorname{deg}_{Q_{n}^{\prime}}(x)$.

By Theorem 6, we have the following corollary.

Corollary 4. In $Q_{n}(n \geqslant 4)$, let $E^{\prime} \subset E\left(Q_{n}\right)$, where $Q_{n}^{\prime}=$ $Q_{n}-E^{\prime}$. If $\min \left\{\operatorname{deg}_{Q_{n}^{\prime}}(v) \mid v \in V\right\}=r \geqslant 4$, then $Q_{n}^{\prime}$ is strong locally r-diagnosable at each node.

\section{Conclusions}

For a system, if we consider only its global properties and ignore its local status, we may lose some interesting and important information. This paper proposes the concept of SLD and derives some conditions for testing the SLD of a node in a system. Moreover, we discuss the relationship between the SLD and the SD of a system. Based on this 
relationship, we obtain the SD of a system by determining the SLD of every node in the system. Our results show that the SLD of a node in $Q_{n}$ is $n$, where $n \geqslant 4$. Then, we consider the SLD of an incomplete hypercube network $Q_{n}-E^{\prime}$, where $E^{\prime} \subset E\left(Q_{n}\right)$. When $0 \leqslant\left|E^{\prime}\right| \leqslant n-3$, the SLD of a node is the same as its RD in $Q_{n}-E^{\prime}$. The above mentioned result still holds in the following two cases: (1) $0 \leqslant\left|E^{\prime}\right| \leqslant 7(n-3)-1$, and for each $v \in V\left(Q_{n}^{\prime}\right), \operatorname{deg}_{Q_{n}^{\prime}}(v) \geqslant 3$. (2) No matter how many edges of $E^{\prime}$ exist, and for each $v \in V\left(Q_{n}^{\prime}\right)$, it follows that $\operatorname{deg}_{Q_{n}^{\prime}}(v)>3$.

It is worth mentioning that haptic identification [25] is an important method of identification, and it is a natural idea to combine haptic identification with the strong local fault identification mentioned by the paper. Our future research work is to explore the strong local haptic identification of a system.

\section{Data Availability}

The data used to support the findings of this study are included within the article

\section{Conflicts of Interest}

The authors declare that there is no conflict of interest regarding the publication of this paper.

\section{Acknowledgments}

This work was supported in part by the Natural Science Foundation of China under Grant no. 61862003 and no. 61761006 and in part by the Natural Science Foundation of the Guangxi Zhuang Autonomous Region of China under Grant no. 2016GXNSFAA380134.

\section{References}

[1] L. C. Ye and J. R. Liang, "Five-round adaptive diagnosis in Hamiltonian networks," IEEE Transactions on Parallel and Distributed Systems, vol. 26, no. 9, pp. 2459-2464, 2015.

[2] Z. Zhao, Y. Liu, and F. Luo, "Output feedback boundary control of an axially moving system with input saturation constraint," ISA Transactions, vol. 68, pp. 22-32, 2017.

[3] J. Fan, X. Jia, X. Liu, S. Zhang, and J. Yu, "Efficient unicast in bijective connection networks with the restricted faulty node set," Information Sciences, vol. 181, no. 11, pp. 23032315, 2011.

[4] J. Fan and X. Jia, "Edge-pancyclicity and path-embeddability of bijective connection graphs," Information Sciences, vol. 178, no. 2, pp. 340-351, 2008.

[5] X. Li, J. Fan, C. K. Lin, and X. Jia, "Diagnosability evaluation of the data center network DCell," Computer Journal, vol. 61, no. 1, pp. 129-143, 2018.

[6] W. Yang and H. Lin, "Reliability evaluation of BC networks in terms of the extra vertex and edge-connectivity," IEEE Transactions on Computers, vol. 63, no. 10, pp. 2540-2548, 2014.

[7] W. Yang, H. Li, and X. Guo, "A kind of conditional fault tolerance of $(n, k)$-star graphs," Information Processing Letters, vol. 110, no. 22, pp. 1007-1011, 2010.
[8] J. Fan and X. Lin, "The t/k-diagnosability of the BC graphs," IEEE Transactions on Computers, vol. 54, no. 2, pp. 176-184, 2005.

[9] C. Yang, Y. Jiang, W. He, J. Na, Z. Li, and B. Xu, "Adaptive parameter estimation and control design for robot manipulators with finite-time convergence," IEEE Transactions on Industrial Electronics, vol. 65, no. 10, pp. 8112-8123, 2018.

[10] Y. Saad and M. H. Schultz, "Topological properties of hypercubes," IEEE Transactions on Computers, vol. 37, no. 7, pp. 867-872, 1988.

[11] F. P. Preparata, G. Metze, and R. T. Chien, "On the connection assignment problem of diagnosable systems," IEEE Transactions on Electronic Computers, vol. EC-16, no. 6, pp. 848854, 1967.

[12] A. Sengupta and A. T. Dahbura, "On self-diagnosable multiprocessor systems: diagnosis by the comparison approach," IEEE Transactions on Computers, vol. 41, no. 11, pp. 13861396, 1992.

[13] C. F. Chiang and J. J. M. Tan, "Using node diagnosability to determine t-diagnosability under the comparison diagnosis model," IEEE Transactions on Computers, vol. 58, no. 2, pp. 251-259, 2009.

[14] P.-L. Lai, J. J. M. Tan, C.-P. Chang, and L.-H. Hsu, "Conditional diagnosability measures for large multiprocessor systems," IEEE Transactions on Computers, vol. 54, no. 2, pp. 165-175, 2005.

[15] S. L. Hakimi and A. T. Amin, "Characterization of connection assignment of diagnosable systems," IEEE Transactions on Computers, vol. C-23, no. 1, pp. 86-88, 1974.

[16] A. Kavianpour and K. H. Kim, "Diagnosabilities of hypercubes under the pessimistic one-step diagnosis strategy," IEEE Transactions on Computers, vol. 40, no. 2, pp. 232-237, 1991.

[17] Z. Zhao, X. Wang, C. Zhang, Z. Liu, and J. Yang, "Neural network based boundary control of a vibrating string system with input deadzone," Neurocomputing, vol. 275, pp. 10211027, 2018.

[18] L.-C. Ye, J.-R. Liang, and H.-X. Lin, "A fast pessimistic diagnosis algorithm for hypercube-like networks under the comparison model," IEEE Transactions on Computers, vol. 65, no. 9, pp. 2884-2888, 2016.

[19] W. Yang, H. Lin, and C. Qin, "On the $t / k$-diagnosability of BC networks," Applied Mathematics and Computation, vol. 225, no. 12 , pp. 366-371, 2013.

[20] G. H. Hsu and J. J. M. Tan, "A local diagnosability measure for multiprocessor systems," IEEE Transactions on Parallel and Distributed Systems, vol. 18, no. 5, pp. 598-607, 2007.

[21] D. Wang, "The diagnosability of hypercubes with arbitrarily missing links," Journal of Systems Architecture, vol. 46, no. 6, pp. 519-527, 2000.

[22] D. West, Introduction to Graph Theory, Prentice Hall, 2001.

[23] A. Dahbura and G. Masson, "An $0\left(n^{2.5}\right)$ fault identification algorithm for diagnosable systems," IEEE Transactions on Computers, vol. C-33, no. 6, pp. 486-492, 1984.

[24] P. K. K. Loh, W. J. Hsu, and Y. Pan, "The exchanged hypercube," IEEE Transactions on Parallel and Distributed Systems, vol. 16, no. 9, pp. 866-874, 2005.

[25] C. Yang, K. Huang, H. Cheng, Y. Li, and C.-Y. Su, "Haptic identification by ELM-controlled uncertain manipulator," IEEE Transactions on Systems, Man, and Cybernetics: Systems, vol. 47, no. 8, pp. 2398-2409, 2017. 


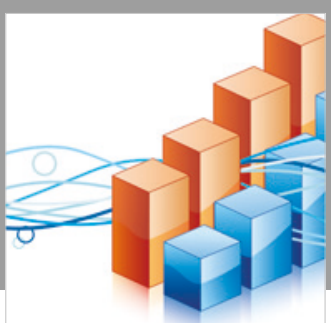

Advances in

Operations Research

\section{-n-m}
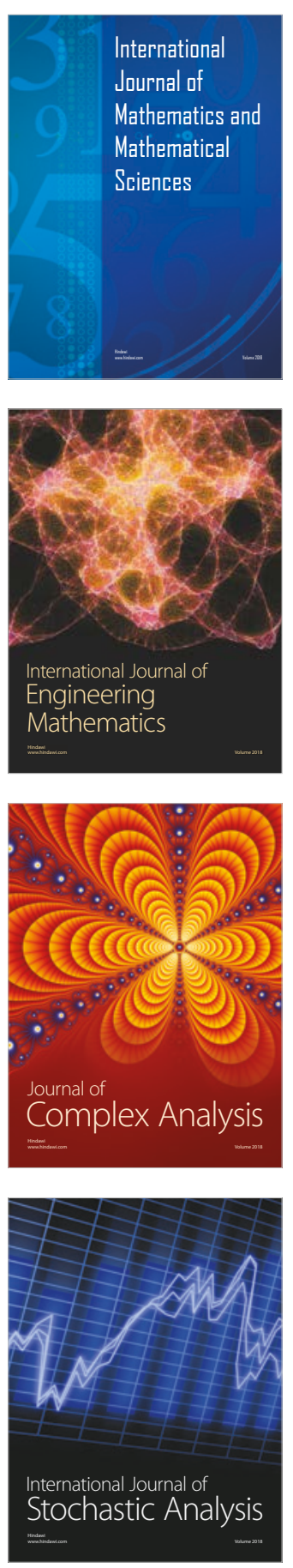
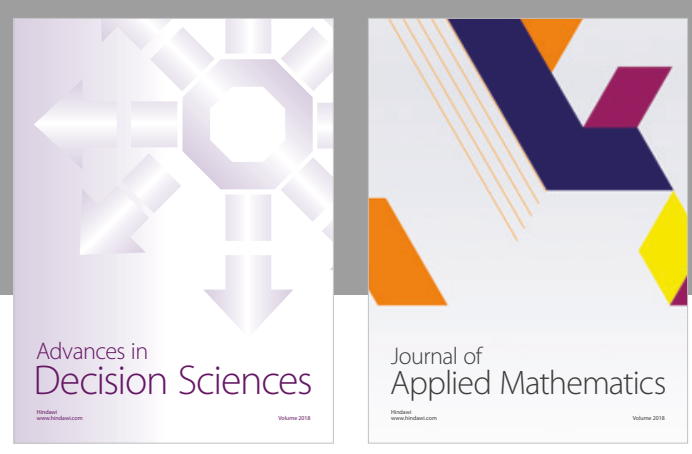

Journal of

Applied Mathematics
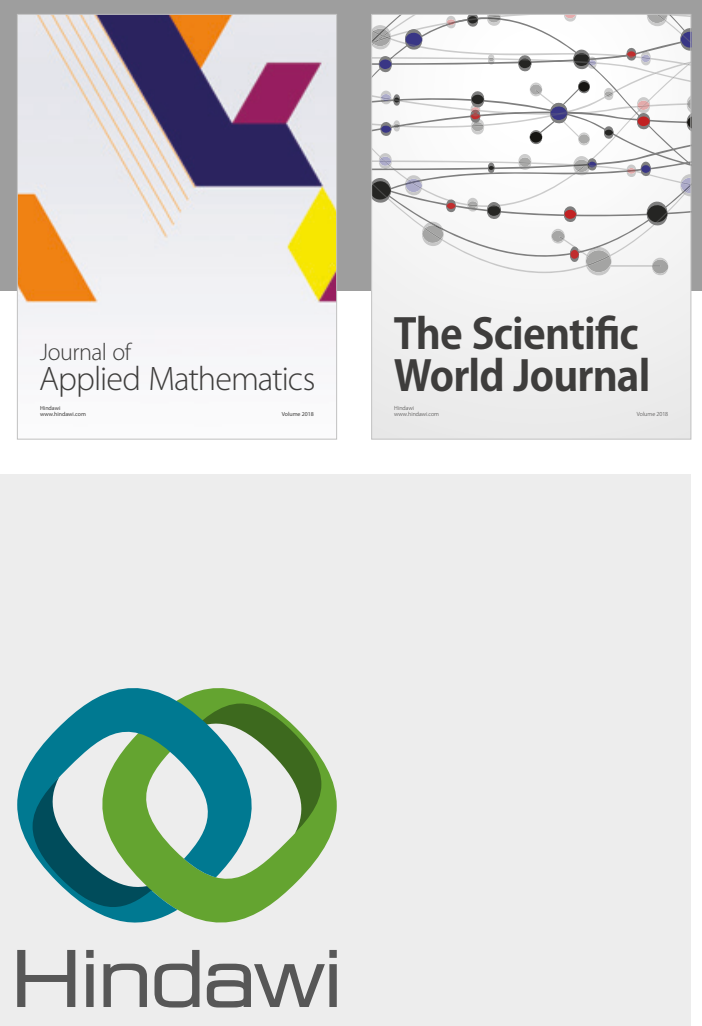

Submit your manuscripts at

www.hindawi.com

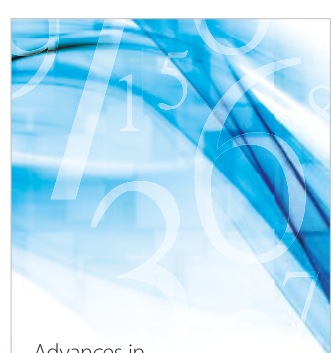

Advances in
Numerical Analysis
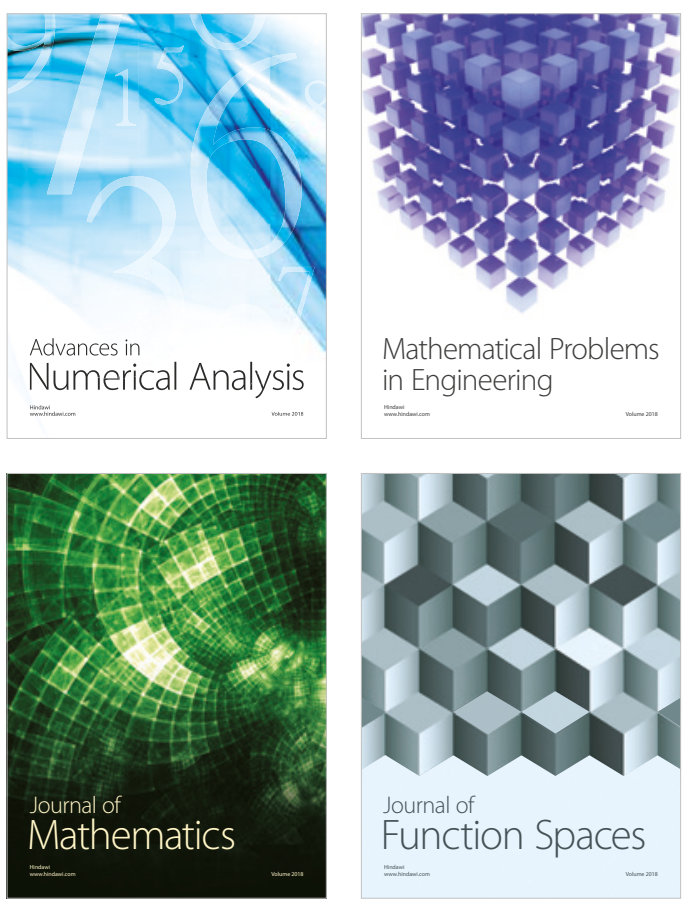

Mathematical Problems in Engineering

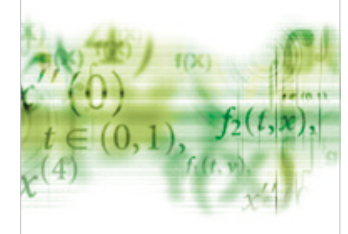

International Journal of

Differential Equations

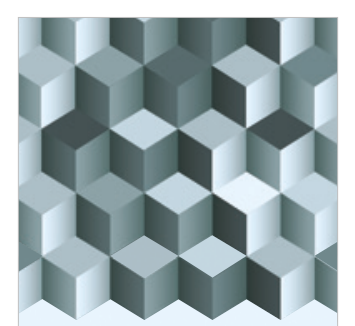

Journal of

Function Spaces
The Scientific

World Journal

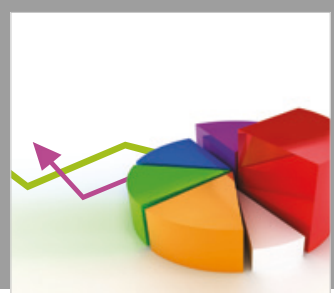

Journal of

Probability and Statistics
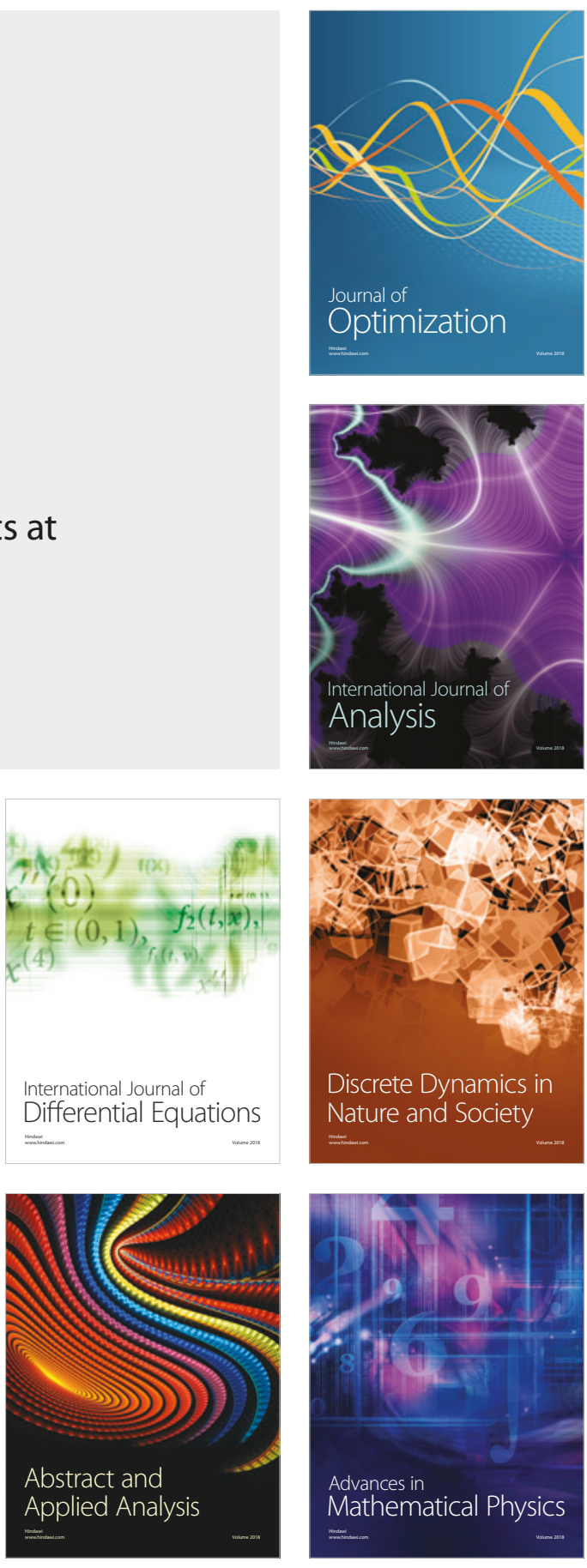